\title{
Boundary Quantum Field Theory on the Interior of the Lorentz Hyperboloid ${ }^{\star}$
}

\author{
Roberto Longo ${ }^{1}$, Karl-Henning Rehren ${ }^{2,3, \star \star}$ \\ 1 Dipartimento di Matematica, Università di Roma "Tor Vergata", Via della Ricerca Scientifica, 1, \\ 00133 Roma, Italy. E-mail: longo@mat.uniroma2.it \\ 2 Institut für Theoretische Physik, Universität Göttingen, Friedrich-Hund-Platz 1, 37077 Göttingen, \\ Germany. E-mail: rehren@ theorie.physik.uni-goettingen.de \\ 3 Courant Research Centre, Higher Order Structures in Mathematics, Bunsenstr. 3-5, 37073 Göttingen, \\ Germany.
}

Received: 7 March 2011 / Accepted: 18 May 2011

Published online: 18 January 2012 - ( ) The Author(s) 2012. This article is published with open access at Springerlink.com

\begin{abstract}
We construct local, boost covariant boundary QFT nets of von Neumann algebras on the interior of the Lorentz hyperboloid $\mathfrak{H}_{R}, x^{2}-t^{2}>R^{2}, x>0$, in the two-dimensional Minkowski spacetime. Our first construction is canonical, starting with a local conformal net on $\mathbb{R}$, and is analogous to our previous construction of local boundary CFT nets on the Minkowski half-space. This net is in a thermal state at Hawking temperature. Then, inspired by a recent construction by E. Witten and one of us, we consider a unitary semigroup that we use to build up infinitely many nets. Surprisingly, the one-particle semigroup is again isomorphic to the semigroup of symmetric inner functions of the disk. In particular, by considering the $U(1)$-current net, we can associate with any given symmetric inner function a local, boundary QFT net on $\mathfrak{H}_{R}$. By considering different states, we shall also have nets in a ground state, rather than in a KMS state.
\end{abstract}

\section{Introduction}

An algebraic description of Boundary CFT on the two-dimensional Minkowski halfspace $x>0$ has been given in [11]. Recently an infinite new family of Boundary QFT on the half-space has been set up in [13] by modifying this construction by elements of a unitary semigroup, a construction conceptually related to the inverse scattering method, in the sense that our first models are associated with (symmetric) scattering functions.

In two dimensions, since locally every Lorentz geometry is conformally flat, boundaries in Minkowski spacetime may be regarded as an analogue of curvature and horizons

* Supported by the ERC Advanced Grant 227458 OACFT "Operator Algebras and Conformal Field Theory", PRIN-MIUR, GNAMPA-INDAM and EU network "Noncommutative Geometry" MRTN-CT-20060031962.

$\star \star$ Supported in part by the German Research Foundation (Deutsche Forschungsgemeinschaft (DFG)) through the Institutional Strategy of the University of Göttingen. 
in higher dimensions. Considering QFT with and without boundaries therefore is a testing ground for the generally covariant locality principle [4], a new paradigm for local quantum field theory stipulating a simultaneous definition on flat and curved spacetimes.

As pointed out to us by Edward Witten, the same question arises in open string theory, notably in the context of gauge-gravity (open-closed) duality. In his words, "studying all possible extensions to manifolds with boundary of a specific $2 \mathrm{~d}$ theory defined initially on manifolds without boundary is the analog of studying Yang-Mills theory in the background of a specified solution of Einstein's equations." His suggestion that this is a question the algebraic approach to QFT might shed light on, had motivated a previous work [13].

In this paper we then study local, relativistic boundary CFT and QFT on the interior $\mathfrak{H}_{R}$ of the Lorentz hyperboloid $x^{2}-t^{2}=R^{2}(x>0)$, in the Minkowski plane. For simplicity we put $R=1$ in this section.

A two-dimensional CFT on $\mathfrak{H}_{R}$ is a local QFT with a conserved and traceless stressenergy tensor, subject to a boundary condition at the boundary $x^{2}-t^{2}=1$. As is well known, conservation and vanishing of the trace imply that the components $T_{L}=$ $\frac{1}{2}\left(T_{00}+T_{01}\right)$ and $T_{R}=\frac{1}{2}\left(T_{00}-T_{01}\right)$ are chiral fields, $T_{L}(u), T_{R}(-v)$, where we are using light-cone coordinates $u=x+t, v=x-t$. The boundary condition is the vanishing of energy flux across the boundary, $T^{0 \mu} \epsilon_{\mu \nu} d x^{\nu}=0$, which in components becomes $(t+x) T_{L}(t+x)=(t-x) T_{R}(t-x)$, namely $\left.u T_{L}(u)\right|_{u v=1}=-\left.v T_{R}(-v)\right|_{u v=1}$, so

$$
u T_{L}(u)=-\frac{1}{u} T_{R}\left(-\frac{1}{u}\right) \equiv T(u)
$$

It follows that the components $T_{10}=T_{01}, T_{11}=T_{00}$ of the stress-energy tensor are of the form

$$
T_{00}(u, v)=\frac{1}{u} T(u)-v T\left(-\frac{1}{v}\right), \quad T_{01}(u, v)=\frac{1}{u} T(u)+v T\left(-\frac{1}{v}\right),
$$

i.e., bi-local expressions in terms of the chiral field $T$.

In terms of the local von Neumann algebras $\mathcal{A}(\mathcal{O})$ generated by the stress-energy tensor, this means that if $I, J$ are bounded intervals of $\mathbb{R}^{+}$with $\mathcal{O}=I \times J$ a double cone contained in $\mathfrak{H}_{R}$ (thus $u v>1 ; u \in I, v \in J$ ) we have

$$
\mathcal{A}(\mathcal{O})=\mathcal{A}_{0}(I) \vee \mathcal{A}_{0}\left(J^{-1}\right)
$$

where $\mathcal{A}_{0}$ is the net on $\mathbb{R}$ generated by the chiral stress-energy tensor (Virasoro net). Indeed only the restriction $\left.\mathcal{A}_{0}\right|_{\mathbb{R}^{+}}$of $\mathcal{A}_{0}$ to the positive half-line enters here.

Dilation covariance of $\left.\mathcal{A}_{0}\right|_{\mathbb{R}^{+}}$gives boost covariance of $\mathcal{A}$ and the KMS property of the vacuum state on $\mathcal{A}_{0}\left(\mathbb{R}^{+}\right)$(Bisognano-Wichmann property) gives the KMS property of the vacuum state on $\mathcal{A}\left(\mathfrak{H}_{R}\right)$ w.r.t. the boosts at Hawking-Unruh inverse temperature $\beta=2 \pi$.

In more generality, starting with any dilation covariant local net $\mathcal{A}_{0}$ of von Neumann algebras on $\mathbb{R}^{+}$we may associate by formula (1.2) a local boost covariant net of von Neumann algebras on $\mathfrak{H}_{R}$. Of course, we could also start with a local translation covariant net on $\mathbb{R}$ as there is a one-to-one correspondence between local translation covariant nets on $\mathbb{R}$ and local dilation covariant nets on $\mathbb{R}^{+}$by the change of variable $x \leftrightarrow e^{x}$. 
Now we can extend the above canonical construction (1.2) based on two local dilation covariant nets $\mathcal{A}_{0}$ and $\mathcal{B}_{0}$ on $\mathbb{R}^{+}$such that $\mathcal{B}_{0}$ is forwardly local w.r.t. $\mathcal{B}_{0}$, i.e., $\mathcal{A}_{0}\left(I_{1}\right)$ commutes with $\mathcal{B}_{0}\left(I_{2}\right)$ if $I_{2}>I_{1}$ :

$$
\mathcal{A}(\mathcal{O})=\mathcal{B}_{0}(I) \vee \mathcal{A}_{0}\left(J^{-1}\right),
$$

where $J^{-1}=\{1 / v: v \in J\}$. The option to have different $\mathcal{A}_{0}$ and $\mathcal{B}_{0}$ is interesting not least in string theory, because the "heterotic string" is of that kind (different internal symmetry groups for left- and right-moving modes, but equal central charge; hence $\mathcal{A}_{0}$ and $\mathcal{B}_{0}$ share a common subnet of the stress-energy tensor). They are both conformal, but not related by a unitary.

However, in analogy with the model building in [13], we may start with a local dilation covariant net $\mathcal{A}_{0}$ and consider the semigroup $\mathcal{E}_{\delta}\left(\mathcal{A}_{0}\right)$ of unitaries $V$ commuting with dilations such that

$$
V \mathcal{A}_{0}(1, \infty) V^{*} \subset \mathcal{A}_{0}(1, \infty) .
$$

Thus $V \mathcal{A}_{0}(a, \infty) V^{*} \subset \mathcal{A}_{0}(a, \infty)$ for all $a>0$. Every element $V \in \mathcal{E}_{\delta}\left(\mathcal{A}_{0}\right)$ then gives a dilation covariant net $\mathcal{B}_{0}=V \mathcal{A}_{0} V^{*}$ that is forwardly local w.r.t. $\mathcal{A}_{0}$, thus a local, boost covariant, Boundary QFT net of von Neumann algebras on $\mathfrak{H}_{R}$ by the formula

$$
\mathcal{A}(\mathcal{O})=V \mathcal{A}_{0}(I) V^{*} \vee \mathcal{A}_{0}\left(J^{-1}\right) .
$$

At this point we may produce families of local, boost covariant nets on $\mathfrak{H}_{R}$ once we compute non-trivial elements of $\mathcal{E}_{\delta}\left(\mathcal{A}_{0}\right)$ for a given local, Möbius covariant net on $\mathbb{R}$.

We shall study the semigroup $\mathcal{E}_{\delta}\left(\mathcal{A}^{0}\right)$ with $\mathcal{A}^{0}$ the $U(1)$-current net on $\mathbb{R}$. One of our main results is the computation of the sub-semigroup of $\mathcal{E}_{\delta}\left(\mathcal{A}^{0}\right)$ consisting of second quantization unitaries (unitaries $V$ that are promotions of one-particle unitaries $V_{0}$ ); we have

$$
\begin{aligned}
& V=\Gamma\left(V_{0}\right) \in \mathcal{E}_{\delta}\left(\mathcal{A}^{0}\right) \Leftrightarrow V_{0}=\varphi(K), \varphi \text { symmetric inner function } \\
& \text { on the upper half-plane, }
\end{aligned}
$$

where $K$ is the one-particle generator of the dilation semigroup.

Thus, rather surprisingly, the above sub-semigroup is naturally isomorphic to the semigroup of inner functions as was the case for the Boundary QFT on the half-space, where positivity of the energy played a crucial role; that role is here played by the KMS thermal equilibrium property.

\section{Basic Definitions}

Let $M$ be the two-dimensional Minkowski spacetime and fix $R>0$. We shall consider the spacetime $\mathfrak{H}_{R}=\left\{(t, x) \in M: x^{2}-t^{2}>R^{2}, x>0\right\}$, the interior of the Lorentz hyperboloid $x^{2}-t^{2}=R^{2}, x>0$. Then $\mathfrak{H}_{R}$ inherits the Lorentz metric from $M$.

The Lorentz boosts provide a one-parameter group of diffeomorphisms $\Lambda$ of $\mathfrak{H}_{R}$ :

$$
\Lambda(s)=\left(\begin{array}{cc}
\cosh s & \sinh s \\
\sinh s & \cosh s
\end{array}\right) .
$$

In light-cone coordinates $u=x+t, v=x-t$, the Lorentz hyperboloid is given by $u v=R^{2}$ and $\mathfrak{H}_{R}$ is the region $u v>R^{2}, u>0$. 
We shall denote by $\mathcal{K}$ the set of double cones strictly contained in $\mathfrak{H}_{R}$, namely $\mathcal{O} \in \mathcal{K}$ if $\mathcal{O}=I_{1} \times I_{2}$ with $I_{1}$ and $I_{2}$ bounded intervals of the chiral lines $v=0, u=0$ and $\overline{\mathcal{O}} \subset \mathfrak{H}_{R}:$ so $\mathcal{O}=\left\{(u, v): u \in I_{1}, v \in I_{2}, u v>R^{2}\right\}$ and $u v>R^{2}$ for all $u \in \bar{I}_{1}$, $v \in \bar{I}_{2}$.

A local net $\mathcal{A}$ of von Neumann algebras on $\mathfrak{H}_{R}$ is a map

$$
\mathcal{O} \mapsto \mathcal{A}(\mathcal{O})
$$

from $\mathcal{K}$ to the set of von Neumann algebras on a (fixed) Hilbert space $\mathcal{H}$ that satisfies the following properties:

1. Isotony: If $\mathcal{O}_{1}, \mathcal{O}_{2}$ are double cones and $\mathcal{O}_{1} \subset \mathcal{O}_{2}$, then $\mathcal{A}\left(\mathcal{O}_{1}\right) \subset \mathcal{A}\left(\mathcal{O}_{2}\right)$.

2. BOOST INVARIANCE: There is a strongly continuous one-pameter unitary group $U$ on $\mathcal{H}$ such that $U(s) \mathcal{A}(\mathcal{O}) U(s)^{*}=\mathcal{A}(\Lambda(s) \mathcal{O}), t \in \mathbb{R}, \mathcal{O} \in \mathcal{K}$.

3. LOCALITY: If $\mathcal{O}_{1}, \mathcal{O}_{2} \in \mathcal{K}$ are spacelike separated, the von Neumann algebras $\mathcal{A}\left(\mathcal{O}_{1}\right)$ and $\mathcal{A}\left(\mathcal{O}_{2}\right)$ commute.

4. INVARIANT STATE: There exists a unit $U$-invariant vector $\xi$, cyclic for $\mathcal{A}\left(\mathfrak{H}_{R}\right) \equiv$ $\bigvee_{\mathcal{O} \in \mathcal{K}} \mathcal{A}(\mathcal{O})$.

If the generator of the one-parameter group is positive, we shall say that $\xi$ is a vacuum vector, the state $(\xi, \cdot \xi)$ a vacuum (equivalently: ground) state, and that the net is in a vacuum (equivalently: ground) representation.

If the one-parameter automorphism group $\tau=\operatorname{Ad} U$ of $\mathcal{A}\left(\mathfrak{H}_{R}\right)$ satisfies the KMS condition w.r.t. $(\xi, \cdot \xi)$, we shall say that $\mathcal{A}$ is in a KMS representation.

2.1. Translation and dilation covariant nets on $\mathbb{R}$. A local, translation (resp. dilation) covariant net of von Neumann algebras on $\mathbb{R}$ (resp. on $\mathbb{R}^{+}$) on a Hilbert space $\mathcal{H}$ is a triple $\left(\mathcal{A}_{0}, U, \xi\right)$ where

- $\mathcal{A}_{0}$ is an isotonous map

$$
I \mapsto \mathcal{A}_{0}(I),
$$

where $I \in \mathcal{I}$ (resp. $I \in \mathcal{I}_{+}$) and $\mathcal{A}_{0}(I)$ is a von Neumann algebra on $\mathcal{H}$;

- $U$ is a unitary one-parameter group on $\mathcal{H}$ such that

$$
U(t) \mathcal{A}_{0}(I) U(-t)=\mathcal{A}_{0}(I+t),\left(\operatorname{resp} . \mathcal{A}_{0}\left(e^{t} I\right)\right), \quad \forall I \in \mathcal{I}\left(\operatorname{resp} . \mathcal{I}_{+}\right), t \in \mathbb{R} ;
$$

- $\xi \in \mathcal{H}$ is a unit $U$-invariant vector, cyclic for $\bigcup_{I} \mathcal{A}_{0}(I)$;

- $\mathcal{A}_{0}\left(I_{1}\right)$ and $\mathcal{A}_{0}\left(I_{2}\right)$ commute if $I_{1}, I_{2}$ are disjoint intervals.

Here $\mathcal{I}\left(\operatorname{resp} \mathcal{I}_{+}\right.$) is the family of bounded, open, non-empty intervals of $\mathbb{R}$ (resp of $\mathbb{R}^{+}$ with $0 \notin \bar{I}$ ). We do not assume positivity of the energy nor irreducibility of the net. If $I_{1}$ is unbounded, $\mathcal{A}_{0}\left(I_{1}\right)$ denotes the von Neumann algebra generated by $\mathcal{A}_{0}(I)$ as $I \subset I_{1}$ runs in the bounded intervals.

If the last condition is not satisfied, the net is called nonlocal.

Note that there is a one-to-one correspondence

$$
\begin{aligned}
& \text { translation covariant nets on } \mathbb{R} \\
& \uparrow \\
& \text { dilation covariant nets on } \mathbb{R}^{+}
\end{aligned}
$$

simply by the "change of variable" $x \leftrightarrow e^{x}$. 
We shall say that the translation covariant nets $\mathcal{A}$ and $\mathcal{A}_{1}$ are abstractly isomorphic (resp. isomorphic) if there is a coherent family of isomorphisms $\Phi_{I}: \mathcal{A}_{1}(I) \rightarrow \mathcal{A}(I)$, $I \in \mathcal{I}$, interchanging the translation action (and the invariant state). Analogous notions can be given for dilation covariant nets.

Let $\mathcal{A}$ be a local Möbius covariant net of von Neumann algebras on $\mathbb{R}$. Denote by $U$ (resp. $V$ ) the one-parameter unitary translation (resp. dilation) group on $\mathcal{H}$. Then $\mathcal{A}$ is a translation covariant net on $\mathbb{R}$, and the restriction of $\mathcal{A}$ to $\mathbb{R}^{+}$is a local dilation covariant net on $\mathbb{R}^{+}$(w.r.t. the vacuum vector). We recall the following fact from [5]:

Proposition 2.1. If $\mathcal{A}_{0}$ is a diffeomorphism covariant local net on $\mathbb{R}$ and $\mathcal{A}_{1}$ the translation covariant net on $\mathbb{R}$ associated with $\left.\mathcal{A}_{0}\right|_{\mathbb{R}^{+}}$by the above correspondence, then $\mathcal{A}_{1}$ and $\mathcal{A}_{0}$ are (canonically) abstractly isomorphic as translation covariant nets.

Therefore one can carry the vacuum state for $\left.\mathcal{A}_{0}\right|_{\mathbb{R}^{+}}$to a translation invariant state for $\mathcal{A}_{0}$. This is the geometric KMS state, a canonical KMS state for $\mathcal{A}_{0}$.

2.2. Forwardly local chiral nets. Let $\mathcal{A}_{0}$ and $\mathcal{B}_{0}$ be local nets of von Neumann algebras on $\mathbb{R}$ on the same Hilbert space. We shall also assume that both $\mathcal{A}_{0}$ and $\mathcal{B}_{0}$ are covariant w.r.t. the same one-parameter translation group. We shall say that $\mathcal{B}_{0}$ is forwardly local w.r.t. $\mathcal{A}_{0}$ if $\mathcal{A}_{0}\left(I_{1}\right)$ commutes with $\mathcal{B}_{0}\left(I_{2}\right)$ for all intervals $I_{1}, I_{2}$ of $\mathbb{R}$ such that $I_{2}>I_{1}$ ( $I_{2}$ is in the future of $I_{1}$ ). We define backwardly local in the obvious way $\left(I_{2}<I_{1}\right)$. $\mathcal{B}_{0}$ is relatively local w.r.t. $\mathcal{A}_{0}$ if it is both forwardly and backwardly local.

We shall say that duality for half-lines holds for $\mathcal{A}_{0}$ if

$$
\mathcal{A}_{0}(I)^{\prime} \cap \mathcal{A}_{0}(\mathbb{R})=\mathcal{A}\left(I^{\prime}\right),
$$

where $I \subset \mathbb{R}$ is any half-line. The following is immediate.

Lemma 2.2. If $\mathcal{B}_{0}$ is forwardly local w.r.t. $\mathcal{A}_{0}$ and duality for half-lines holds for $\mathcal{A}_{0}$, then $\mathcal{B}_{0}(a, \infty) \subset \mathcal{A}_{0}(a, \infty), a \in \mathbb{R}$.

Proof. $\mathcal{B}_{0}(a, \infty)$ commutes with $\mathcal{A}_{0}(-\infty, a)$, so is contained in $\mathcal{A}_{0}(-\infty, a)^{\prime} \cap \mathcal{A}_{0}(\mathbb{R})=$ $\mathcal{A}_{0}(a, \infty)$.

If duality for half-lines holds for both $\mathcal{A}_{0}$ and $\mathcal{B}_{0}$, and $\mathcal{A}_{0}$ and $\mathcal{B}_{0}$ are relatively local, then $\mathcal{B}_{0}(I)=\mathcal{A}_{0}(I)$ for all half-lines $I \subset \mathbb{R}$.

It is immediate to translate the above notion for local nets on $\mathbb{R}^{+}$by the correspondence (2.1). If $\mathcal{A}_{0}$ is a local Möbius covariant net of von Neumann algebras on $\mathbb{R}$, then duality for half-lines holds for $\left.\mathcal{A}_{0}\right|_{\mathbb{R}^{+}}$(namely $\mathcal{A}_{0}(0, a)^{\prime} \cap \mathcal{A}_{0}(0, \infty)=\mathcal{A}_{0}(a, \infty)$, $a>0$ ) iff $\mathcal{A}_{0}$ is strongly additive.

\section{BQFT on the Interior of the Lorentz Hyperboloid}

Let $\mathcal{A}_{0}$ and $\mathcal{B}_{0}$ be dilation covariant, local nets of von Neumann algebras on $\mathbb{R}^{+}$with $\mathcal{B}_{0}$ forwardly local w.r.t. $\mathcal{A}_{0}$.

Given intervals $I_{1}, I_{2}$ of $\mathbb{R}^{+}$we define the von Neumann algebra $\mathcal{A}(\mathcal{O})$ associated with the double cone $\mathcal{O}=I_{1} \times I_{2}$ by

$$
\mathcal{A}(\mathcal{O})=\mathcal{B}_{0}\left(I_{1}\right) \vee \mathcal{A}_{0}\left(R^{2} I_{2}^{-1}\right)
$$

where $I^{-1} \equiv\left\{\lambda^{-1}: \lambda \in I\right\}$. 
Formally we can write

$$
\mathcal{A}(u, v)=\mathcal{B}_{0}(u) \vee \mathcal{A}_{0}\left(R^{2} / v\right)
$$

and for simplicity we use this formal writing here below; one can easily properly write up by replacing a point $(u, v) \in \mathfrak{H}_{R}$ with a double cone $\mathcal{O} \in \mathcal{K}$.

Theorem 3.1. $\mathcal{A}$ is a local, boost covariant net of von Neumann algebras on $\mathfrak{H}_{R}$.

Proof. To check locality, we have to show that if $(u, v)$ and $\left(u^{\prime}, v^{\prime}\right)$ are points of $\mathfrak{H}_{R}$ that are spacelike, i.e., $\left(u^{\prime}-u\right)\left(v^{\prime}-v\right)>0$, then $\mathcal{A}(u, v)$ and $\mathcal{A}\left(u^{\prime}, v^{\prime}\right)$ commute. So choose $(u, v)$ and $\left(u^{\prime}, v^{\prime}\right)$ with $u v>R^{2}$ and $u^{\prime}>u, v^{\prime}>v$.

Since $u^{\prime}>u>R^{2} / v>R^{2} / v^{\prime}, \mathcal{B}_{0}\left(u^{\prime}\right)$ commutes with $\mathcal{B}_{0}(u)$ (by locality of $\mathcal{B}_{0}$ ) and with $\mathcal{A}_{0}\left(R^{2} / v\right)$ (by forward locality), so $\mathcal{B}_{0}\left(u^{\prime}\right)$ commutes with $\mathcal{A}(u, v)$. Moreover $\mathcal{A}_{0}\left(R^{2} / v^{\prime}\right)$ commutes with $\mathcal{A}_{0}\left(R^{2} / v\right)$ (by locality of $\left.\mathcal{A}_{0}\right)$ and with $\mathcal{B}_{0}(u)$ (by forward locality). Therefore $\mathcal{B}_{0}\left(u^{\prime}\right) \vee \mathcal{A}_{0}\left(R^{2} / v^{\prime}\right)$ commutes with $\mathcal{B}_{0}(u) \vee \mathcal{A}_{0}\left(R^{2} / v\right)$.

Concerning the covariance, let $U$ be the dilation one parameter unitary group of $\mathcal{A}_{0}$ and $\mathcal{B}_{0}$, thus $U(s) \mathcal{B}_{0}(u) U(-s)=\mathcal{B}_{0}\left(e^{s} u\right)$ and $U(s) \mathcal{A}_{0}(v) U(-s)=\mathcal{A}_{0}\left(e^{s} v\right)$. Then

$$
\begin{aligned}
& U(s) \mathcal{A}(u, v) U(-s)=U(s)\left(\mathcal{B}_{0}(u) \vee \mathcal{A}_{0}\left(R^{2} / v\right)\right) U(-s) \\
& \quad=\mathcal{B}_{0}\left(e^{s} u\right) \vee \mathcal{A}_{0}\left(e^{s} R^{2} / v\right)=\mathcal{A}\left(e^{s} u, e^{-s} v\right)=\mathcal{A}(\Lambda(s)(u, v))
\end{aligned}
$$

as desired.

Proposition 3.2. $\mathcal{A}\left(\mathfrak{H}_{R}\right)$ is generated by $\mathcal{B}_{0}\left(\mathbb{R}^{+}\right)$and $\mathcal{A}_{0}\left(\mathbb{R}^{+}\right)$. So $\mathcal{A}\left(\mathfrak{H}_{R}\right)=\mathcal{A}_{0}\left(\mathbb{R}^{+}\right)$if $\mathcal{B}_{0}\left(\mathbb{R}^{+}\right) \subset \mathcal{A}_{0}\left(\mathbb{R}^{+}\right)$, in particular if duality for half-lines holds for $\mathcal{A}_{0}$.

Proof. Immediate.

Corollary 3.3. If $\mathcal{B}_{0}\left(\mathbb{R}^{+}\right) \subset \mathcal{A}_{0}\left(\mathbb{R}^{+}\right)$, $\xi$ is a vacuum (resp. $K M S$ ) vector for $\mathcal{A}$ (w.r.t. the boosts) if it is a vacuum (resp. KMS) vector for $\mathcal{A}_{0}$ (w.r.t. the dilations).

3.1. Constructing $Q F T$ on $\mathfrak{H}_{R}$ by an element of the semigroup $\mathcal{E}_{\delta}(\mathcal{A})$. Let now $\mathcal{A}_{0}$ be a local, dilation covariant net of Neumann algebras on $\mathbb{R}^{+}$. We denote by $\mathcal{E}_{\delta}\left(\mathcal{A}_{0}\right)$ the group of unitaries on $\mathcal{H}$, commuting with the dilation unitary group $U$, such that $V \mathcal{A}_{0}(1, \infty) V^{*} \subset \mathcal{A}_{0}(1, \infty)$. As $V$ commutes with dilations, $V \mathcal{A}_{0}(a, \infty) V^{*} \subset$ $\mathcal{A}_{0}(a, \infty)$ for all $a>0$.

Proposition 3.4. Let $\mathcal{A}_{0}$ be in a KMS representation with an extremal KMS state. Then for each $V \in \mathcal{E}_{\delta}\left(\mathcal{A}_{0}\right)$, one has $V \mathcal{A}_{0}\left(\mathbb{R}^{+}\right) V^{*}=\mathcal{A}_{0}\left(\mathbb{R}^{+}\right)$.

Proof. If the representation arises from an extremal KMS state, $\mathcal{A}_{0}\left(\mathbb{R}^{+}\right)$is a factor. In particular, the GNS vector $\xi$ is (up to phases) the unique dilation invariant vector. Because $V$ commutes with the dilations, $V^{*} \xi$ is equal to $\xi$ up to a phase, therefore cyclic for $\mathcal{A}_{0}\left(\mathbb{R}^{+}\right)$. Thus, $V \mathcal{A}_{0}\left(\mathbb{R}^{+}\right) V^{*}$ is contained in $\mathcal{A}_{0}\left(\mathbb{R}^{+}\right)$, cyclic on the invariant vector $\xi$, and globally invariant under the modular group of $\mathcal{A}_{0}\left(\mathbb{R}^{+}\right)$(the rescaled dilations). So $V \mathcal{A}_{0}\left(\mathbb{R}^{+}\right) V^{*}=\mathcal{A}_{0}\left(\mathbb{R}^{+}\right)$by Takesaki's theorem. 
If $\mathcal{A}_{0}$ is a local, Möbius covariant net of Neumann algebras on $\mathbb{R}$, then the restriction $\left.\mathcal{A}_{0}\right|_{\mathbb{R}^{+}}$of $\mathcal{A}_{0}$ to $\mathbb{R}^{+}$is a local, dilation covariant net on $\mathbb{R}^{+}$(in a KMS representation) and we simply set $\mathcal{E}_{\delta}\left(\mathcal{A}_{0}\right)=\mathcal{E}_{\delta}\left(\left.\mathcal{A}_{0}\right|_{\mathbb{R}^{+}}\right)$.

Setting

$$
\mathcal{B}_{0}(I)=V \mathcal{A}_{0}(I) V^{*}, \quad I \in \mathcal{I}_{+},
$$

$\mathcal{B}_{0}$ is a local net of von Neumann algebras on $\mathbb{R}_{+}$, with dilation unitary group $U$. The net $\mathcal{B}_{0}$ is forwardly local w.r.t. $\mathcal{A}_{0}$ : if $0<a<b<c<d$ we have $\mathcal{B}_{0}(c, d) \subset \mathcal{B}_{0}(c, \infty) \subset$ $\mathcal{A}_{0}(c, \infty)$ so $\mathcal{B}_{0}(c, d)$ commutes with $\mathcal{A}_{0}(a, b)$.

With $V$ a unitary in $\mathcal{E}_{\delta}\left(\mathcal{A}_{0}\right)$ we denote by $\mathcal{A}_{0 V}$ the local net on $\mathfrak{H}_{R}$ associated with $\mathcal{A}_{0}$ and $\mathcal{B}_{0}=V \mathcal{A}_{0} V^{*}$, i.e.,

$$
\mathcal{A}_{0 V}(\mathcal{O})=V \mathcal{A}_{0}\left(I_{1}\right) V^{*} \vee \mathcal{A}_{0}\left(I_{2}^{-1}\right),
$$

where $\mathcal{O}=I_{1} \times I_{2} \in \mathcal{K}$. So we have:

Proposition 3.5. $\mathcal{A}_{0 V}$ is a local, boost covariant net of von Neumann algebras on $\mathfrak{H}_{R}$.

By Cor. 3.3, $\mathcal{A}_{0 V}$ is in a vacuum (resp. KMS) representation w.r.t. the boosts if $\left.\mathcal{A}_{0}\right|_{\mathbb{R}^{+}}$ is in a vacuum (resp. KMS) representation w.r.t. the dilations.

So, in particular, given a local Möbius covariant net $\mathcal{A}$ on $\mathbb{R}$, we have maps:

$$
V \in \mathcal{E}\left(\mathcal{A}_{0}\right) \mapsto \mathrm{BQFT} \text { net } \mathcal{A}_{0 V} \text { on } \mathfrak{H}_{R} \text { in a ground representation }
$$

and

$$
V \in \mathcal{E}_{\delta}\left(\mathcal{A}_{0}\right) \mapsto \text { BQFT net } \mathcal{A}_{0 V} \text { on } \mathfrak{H}_{R} \text { in a KMS representation, }
$$

where the inverse temperature is $\beta=2 \pi$. Here, as in [13], $\mathcal{E}\left(\mathcal{A}_{0}\right)$ is the semigroup of unitaries commuting with translations such that $V \mathcal{A}_{0}\left(\mathbb{R}^{+}\right) V^{*} \subset V \mathcal{A}_{0}\left(\mathbb{R}^{+}\right) V^{*}$.

We shall say that two nets $\mathcal{B}_{1}, \mathcal{B}_{2}$ on $\mathfrak{H}_{R}$, acting on the Hilbert spaces $\mathcal{H}_{1}$ and $\mathcal{H}_{2}$, are locally isomorphic if for every double cone $\mathcal{O} \in \mathcal{K}$ there is an isomorphism $\Phi_{\mathcal{O}}: \mathcal{B}_{1}(\mathcal{O}) \rightarrow \mathcal{B}_{2}(\mathcal{O})$ such that

$$
\Phi_{\tilde{\mathcal{O}}} \mid \mathcal{B}_{1}(\mathcal{O})=\Phi_{\mathcal{O}}
$$

if $\mathcal{O}, \tilde{\mathcal{O}} \in \mathcal{K}, \mathcal{O} \subset \tilde{\mathcal{O}}$ and

$$
U_{2}(t) \Phi_{\mathcal{O}}(X) U_{2}(-t)=\Phi_{\mathcal{O}+t}\left(U_{1}(t) X U_{1}(-t)\right), \quad X \in \mathcal{B}_{1}(\mathcal{O})
$$

with $U_{1}$ and $U_{2}$ the corresponding boost unitary groups on $\mathcal{H}_{1}$ and $\mathcal{H}_{2}$.

Proposition 3.6. Let $\mathcal{A}_{0}$ be a local Möbius covariant net of Neumann algebras on $\mathbb{R}$ with the split property. If $V$ and $W$ are unitaries in $\mathcal{E}_{\delta}(\mathcal{A})$ the nets $\mathcal{A}_{0 V}$ and $\mathcal{A}_{0 W}$ on $\mathfrak{H}_{R}$ are locally isomorphic.

Proof. The proof is similar to the one for the case of Boundary QFT on the half-space [13, Prop. 3.5]. 
3.2. Induced nets on $M_{+}$and on $\mathfrak{H}_{R}$. The logarithmic map $t+x:=\log u, t-x:=$ $\log R^{2} / v$ is a diffeomorphism of the hyperboloid $\mathfrak{H}_{R}$ to the Minkowski half-space $M_{+}=$ $\left\{(t, x) \in \mathbb{R}^{2}: x>0\right\}$. We write $I \times J:=\{(t, x): t+x \in I, t-x \in J\}$. Then $I \times J \subset M_{+}$ iff $I>J$. The diffeomorphism identifies double cones $I \times J \subset M_{+}$with double cones $e^{I} \times R^{2} e^{-J} \subset \mathfrak{H}_{R}$.

Let $\mathcal{A}(I)=\mathcal{A}_{0}\left(e^{I}\right)$ and $\mathcal{B}(I)=\mathcal{B}_{0}\left(e^{I}\right)$ be the translation covariant nets on $\mathbb{R}$ associated with $\left.\mathcal{A}_{0}\right|_{\mathbb{R}^{+}}$and $\left.\mathcal{B}_{0}\right|_{\mathbb{R}^{+}}$by the logarithmic map. We use the diffeomorphism to transfer the above net (3.1) on $\mathfrak{H}_{R}$ to a net on $M_{+}$, namely $\mathcal{A}_{+}(I \times J):=\mathcal{A}_{\mathfrak{H}_{R}}\left(e^{I} \times R^{2} e^{-J}\right)=$ $\mathcal{B}_{0}\left(e^{I}\right) \vee \mathcal{A}_{0}\left(e^{J}\right)$ for $I>J$. Thus,

$$
\mathcal{A}_{+}(I \times J)=\mathcal{B}(I) \vee \mathcal{A}(J)
$$

This is a local net on the Minkowski half-space $M_{+}$. If $\mathcal{A}$ and $\mathcal{B}$ are the same (Möbius covariant) local nets, (3.5) is the BCFT net of chiral observables, constructed in [11].

The discussion in the beginning of this section shows that (3.5) is local if $\mathcal{A}$ and $\mathcal{B}$ do not necessarily coincide, but $\mathcal{B}$ is forwardly local w.r.t. $\mathcal{A}$. Indeed, if $I_{1} \times J_{1} \subset M_{+}$ and $I_{2} \times J_{2} \subset M_{+}$are spacelike to each other, then without loss of generality $I_{2}>I_{1}>$ $J_{1}>J_{2}$, so that forwardly locality is necessary and sufficient to establish locality of the net $\mathcal{A}_{+}$.

In [13], local nets $\mathcal{B}$ that are forwardly local w.r.t. a given local net $\mathcal{A}$ are constructed by conjugation with a unitary from the semigroup $\mathcal{E}$. Thus, the present construction comprises the two previous cases as special cases (after mapping the hyperboloid onto the half-space); but the emphasis in this article is on the more flexible choice of states and representations. In particular, we here admit $\mathcal{A}_{0}$ in the vacuum representation, which means that $\mathcal{A}=\left.\mathcal{A}_{0}\right|_{\mathbb{R}^{+}}$is in a KMS representation (see above).

In this subsection, we want to place the present construction into context with the more general construction of non-chiral BCFT observables on the half-space, presented in [11]. Namely, in [11], we have considered a nonlocal but relatively local chiral extension of $\mathcal{A}$, which we call $\mathcal{C}$ here to prevent confusion with $\mathcal{B}$ above; i.e., $\mathcal{A}(I) \subset \mathcal{C}(I)$ for all $I \in \mathcal{I}$. We then define the induced net on $M_{+}$,

$$
\mathcal{C}_{+}(I \times J):=\mathcal{C}(K)^{\prime} \cap \mathcal{C}(L)
$$

where $K$ is the open interval between $I$ and $J$, and $L$ is the open interval whose closure equals the closure of $I \cup K \cup J$ (i.e., $L=I \cup K \cup J$ plus two interior points). $\mathcal{C}_{+}$is a local net on $M_{+}$, and it contains the subnet $\mathcal{A}_{+}(I \times J)=\mathcal{A}(I) \vee \mathcal{A}(J)$ of chiral observables.

Also here, the conditions in [11] can actually be relaxed: Let $\mathcal{A}, \mathcal{B}$ be two local nets of von Neumann algebras on $\mathbb{R}$, and $\mathcal{C}$ a possibly nonlocal net that extends both $\mathcal{A}$ and $\mathcal{B}$.

Proposition 3.7. If $\mathcal{C}$ is forwardly local w.r.t. $\mathcal{A}$ and backwardly local w.r.t. $\mathcal{B}$, then $\mathcal{B}$ is forwardly local w.r.t. $\mathcal{A}$. The induced net $\mathcal{C}_{+}$on the half-space $M_{+}$defined by (3.6) is local and contains $\mathcal{A}_{+}(I \times J)=\mathcal{B}(I) \vee \mathcal{A}(J)$. Moreover, $\mathcal{C}_{+}$is covariant under time translations if $\mathcal{A}, \mathcal{B}$ and $\mathcal{C}$ are translation covariant and the translations of $\mathcal{C}$ restrict to the translations of $\mathcal{A}$ and $\mathcal{B}$. 
There is also a partial converse: given a local net $\mathcal{D}_{+}$on $M_{+}$, one may define associated nets on the boundary $\mathbb{R}$ as follows:

$$
\begin{aligned}
\mathcal{A}(J) & :=\bigcap_{I: I>J} \mathcal{D}_{+}(I \times J), \\
\mathcal{B}(I): & : \bigcap_{J: J<I} \mathcal{D}_{+}(I \times J), \\
\mathcal{C}(L): & : \bigvee_{I, J \subset L: I>J} \mathcal{D}_{+}(I \times J) .
\end{aligned}
$$

The following result is also straightforward.

Proposition 3.8. $\mathcal{C}$ contains both $\mathcal{A}$ and $\mathcal{B}$, and $\mathcal{C}$ is forwardly local w.r.t. $\mathcal{A}$ and backwardly local w.r.t. $\mathcal{B}$. In particular, $\mathcal{B}$ is forwardly local w.r.t. $\mathcal{A}$, and both $\mathcal{A}$ and $\mathcal{B}$ are local. If in addition $\mathcal{D}_{+}$is time translation covariant, then $\mathcal{A}, \mathcal{B}$ and $\mathcal{C}$ are translation covariant and the translations of $\mathcal{C}$ restrict to the translations of $\mathcal{A}$ and $\mathcal{B}$. Moreover, one has the inclusions

$$
\mathcal{B}(I) \vee A(J) \subset \mathcal{D}_{+}(I \times J) \subset \mathcal{C}(K)^{\prime} \cap \mathcal{C}(L) .
$$

In particular, every local net on $M_{+}$is intermediate between a net of the form (3.5) and an induced net of the form (3.6).

Proof. Only the locality properties need a little argument. If $L>J$, choose $I>L$. Then $I \times J$ is spacelike separated from $I_{1} \times J_{1}$ for all $I_{1}, J_{1} \subset L, I_{1}>J_{1}$. Because $\mathcal{D}_{+}$is local, it follows that $\mathcal{C}(L)$ commutes with $\mathcal{A}(J)$, i.e., $\mathcal{C}$ is forwardly local w.r.t. $\mathcal{A}$. Backward locality of $\mathcal{C}$ w.r.t. $\mathcal{B}$ is similar, and the other statements follow because $\mathcal{C}(I)$ contains $\mathcal{A}(I)$ and $\mathcal{B}(I)$. Notice that a net is local if it is forwardly or backwardly local w.r.t. itself.

It would be interesting to characterize possible nonlocal nets $\mathcal{C}$ for which the induced net is not trivial $\left(=\mathbb{C}\right.$ ). E.g., if $\mathcal{D}_{+}$is given as in (3.5), and $\mathcal{C}$ is generated from $\mathcal{D}_{+}$as in (3.8), then the net induced from $\mathcal{C}$ contains at least $\mathcal{B}(I) \vee \mathcal{A}(J)$. Even if $\mathcal{B}=\mathcal{A}$, the extension $\mathcal{A}(I) \vee \mathcal{A}(J) \subset \mathcal{C}_{+}(I \times J)$ is nontrivial in general, reflecting the superselection sectors of $\mathcal{A}[8,11]$. Thus, for a given local net $\mathcal{A}$ and an element $V$ of the associated semigroup $\mathcal{E}$, putting $\mathcal{B}=V \mathcal{A} V^{*}$, it would be a highly interesting problem to understand the structure of the resulting induced net in terms of $\mathcal{A}$ and $V$.

The analogous question arises when $\mathcal{A}$ and $\mathcal{B}$ are different chiral extensions of a given chiral net (e.g., that of the stress-energy tensor), differing by a cohomological twist of the Q-system [7]. We hope to return to these issues in a future publication.

Clearly, the entire discussion of this subsection can be transferred back to the hyperboloid by the change of coordinates as above. Thus, on the hyperboloid one has for $I, J \in \mathcal{I}_{+}, I>R^{2} J^{-1}$,

$$
C_{\mathfrak{H}_{R}}(I \times J)=C_{0}(K)^{\prime} \cap C_{0}(L),
$$

where $C_{0}$ is a net on $\mathbb{R}^{+}$, and $K$ is the open interval between $I$ and $R^{2} J^{-1}$, and $L=$ $I \cup K \cup R^{2} J^{-1}$ plus two interior points. If $C_{0}$ is dilation covariant, then $C_{\mathfrak{H}_{R}}$ is Lorentz covariant. 


\section{Endomorphisms of Standard Subspaces}

4.1. Preliminary comments. With $a \in(0, \infty]$ we denote by $\mathbb{S}_{a}$ the strip of the complex plane $\{z \in \mathbb{C}: 0<\mathfrak{\Im} z<a\}$ (so $\mathbb{S}_{\infty}$ is the upper half-plane). Let $f \in L^{1}(\mathbb{R})$, and $\varphi$ be the Fourier transform of $f$. Then, if $f$ is real and $\operatorname{supp}(f) \subset \mathbb{R}^{+}, \varphi$ is a symmetric function $(\varphi(-s)=\overline{\varphi(s)})$ and belongs to the Hardy space $\mathbb{H}^{\infty}\left(\mathbb{S}_{\infty}\right)$, namely it is the boundary value of a bounded analytic function on the upper half-plane $\mathbb{S}_{\infty}$. The space of such functions is weakly dense in $\mathbb{H}^{\infty}\left(\mathbb{S}_{\infty}\right)$.

Let $\mathcal{H}$ be a (complex) Hilbert space, $H_{1}$ a real Hilbert subspace of $\mathcal{H}$ and $K$ a selfadjoint operator on $\mathcal{H}$. Suppose that

$$
e^{i t K} H_{1} \subset H_{1}, \quad \forall t \geq 0 .
$$

For $f$ and its Fourier transform $\varphi$ as before, we have

$$
\varphi(K)=\int_{-\infty}^{\infty} f(t) e^{i t K} d t .
$$

Then, if $\operatorname{supp}(f) \subset \mathbb{R}^{+}$, we have $\varphi(K) H_{1} \subset H_{1}$.

Suppose further that $K$ has Lebesgue spectrum. Then the map $\varphi \in L^{\infty}(\mathbb{R}) \mapsto$ $\varphi(K) \in B(\mathcal{H})$ is weakly continuous so, taking limits, we have

$$
\varphi(K) H_{1} \subset H_{1}, \quad \forall \varphi \in \mathbb{H}^{\infty}\left(\mathbb{S}_{\infty}\right), \varphi \text { symmetric, }
$$

and, in particular, every symmetric inner function $\varphi$ on $\mathbb{S}_{\infty}$ gives a unitary $V=\varphi(K)$ such that $V H_{1} \subset H_{1}$.

We shall see here below a situation where this occurs. In our case a converse will hold. Our standard subspace methods will be appropriate as they allow us to prove such a converse too; moreover the reducible case is treatable too.

4.2. Characterization of the semigroup. Let $\mathcal{H}$ be a complex Hilbert space, $H \subset \mathcal{H}$ a standard subspace, i.e., a closed real subspace such that $H+i H$ is dense in $\mathcal{H}$ and $H \cap i H=\{0\}$, and $\Delta_{H}, J_{H}$ the modular operator and modular conjugation of $H$.

Recall that, if $V \in B(\mathcal{H})$ a bounded linear operator on $\mathcal{H}$, we have the following $[1,9]$.

Proposition 4.1. The following are equivalent:

(i) $V H \subset H$;

(ii) The map $s \in \mathbb{R} \rightarrow V(s) \equiv \Delta^{-i s} V \Delta^{i s}$ extends to a bounded weakly continuous function on the closed strip $\overline{\mathbb{S}_{1 / 2}}$, analytic in $\mathbb{S}_{1 / 2}$, such that $V(i / 2)=J V J$,

where $\Delta=\Delta_{H}, J=J_{H}$.

Let now $(H, T)$ be a standard pair of the Hilbert space $\mathcal{H}$. Namely $H$ is a standard subspace of the Hilbert space $\mathcal{H}$ and there exists a one parameter unitary group $T(t)=e^{i t P}$ on $\mathcal{H}$ such that $T(t) H \subset H$ for all $t \geq 0$, and $P>0$. Then

Assume that $(H, T)$ is irreducible and let $H_{a} \equiv T(a) H$, so $\Delta_{H_{1}}=T(1) \Delta T(-1)$.

$$
\Delta^{-i s} H_{1} \subset H_{1}, \quad s \geq 0,
$$

indeed $\Delta^{-i s} H_{1}=\Delta^{-i s} T(1) H=T\left(e^{2 \pi s}\right) \Delta^{-i s} H=T\left(e^{2 \pi s}\right) H=H_{e^{2 \pi s}}$.

If an inclusion of standard subspaces $H_{1} \subset H$ satisfies the condition (4.1) we shall say that $H_{1} \subset H$ is a half-sided modular (hsm) inclusion (of standard subspaces). 
Proposition 4.2. Let $H_{1} \subset H$ be an inclusion of standard subspaces of $\mathcal{H}$. The following are equivalent:

(i) $H_{1} \subset H$ is a half-sided modular inclusion.

(ii) There exists a standard pair $(H, T)$ with $T(1) H=H_{1}$. Moreover $T$ is uniquely determined.

Proof. The equivalence between $(i)$ and ( $i i)$ follows by the analogs of theorems by Borchers and Wiesbrock, see [9], except for the uniqueness of $T$ that we show now. Given the hsm inclusion $H_{1} \subset H$, let $T_{1}$ and $T_{2}$ be one-parameter unitary groups on $\mathcal{H}$ such that $T_{i}(t) H \subset H, t \geq 0$ and $T_{i}(1) H=H_{1}$. Then $T_{2}(-1) T_{1}(1) H=H$, so $T_{2}(-1) T_{1}(1)$ commutes with $\Delta$. On the other hand $\Delta^{-i s} T_{2}(-1) T_{1}(1) \Delta^{i s}=T_{2}\left(-e^{2 \pi s}\right) T_{1}\left(e^{2 \pi s}\right)$, so $T_{2}(-t) T_{1}(t)=T_{2}(-1) T_{1}(1), t \geq 0$; thus $T_{2}(1-t)=T_{1}(1-t)$ and $T_{1}=T_{2}$ by the group property.

Proposition 4.3. Let $H_{1} \subset H$ be an inclusion of standard subspaces of $\mathcal{H}$ and $V$ be a unitary on $\mathcal{H}$ such that $V H_{1} \subset H_{1}$. Then $V$ commutes with $\Delta$ iff $V H=H$.

Proof. If $V H=H$ then $V$ commutes with $\Delta$ by modular theory, see [9]. We show the converse.

As $\Delta^{i s} H_{1}=H_{e^{-2 \pi s}}$ and $V$ commutes with $\Delta$, we have $V H_{a} \subset H_{a}$ for all $a>0$, thus $V H \subset H$ because $H=\overline{\cup_{a>0} H_{a}}$ (as $\overline{\cup_{a>0} H_{a}}$ is a standard $\Delta^{i s}$-invariant subspace of $H$ ).

Now $V H \subset H$ and $V \Delta^{i s}=\Delta^{i s} V$ imply that $V H=H$ because $V H$ is a standard, $\Delta^{i s}$-invariant subspace of $H$.

Lemma 4.4. Let $H_{1} \subset H$ be an inclusion of standard subspaces of $\mathcal{H}$ as in Prop. 4.2. Let $V$ be a unitary on $\mathcal{H}$, commuting with $\Delta$. Then $V=\varphi(K)$, where $2 \pi K=-\log \Delta$ and $\varphi$ is a Borel function on $\mathbb{R}$ with $|\varphi(x)|=1$ for almost all $x \in \mathbb{R}$.

Moreover $V H_{1} \subset H_{1}$ iff the operator-valued function

$$
F: a \in(1, \infty) \mapsto \varphi(K+a P)
$$

admits a bounded analytic continuation in the upper half-plane $\mathbb{S}_{\infty}$, and $\left.F(z)\right|_{z=0}=$ $\varphi(K)$.

Proof. As $(H, T)$ is irreducible and $V$ commutes with $\Delta$, we have $V=\varphi(K)$ with $\varphi$ is a Borel function on $\mathbb{R}$ and $|\varphi(x)|=1$ for almost all $x \in \mathbb{R}$ by the unitarity of $V$.

By Prop. 4.2 $\mathrm{VH}=H$ and in particular

$$
V J=J V .
$$

So we have

$$
\varphi(K)=V=J V J=J \varphi(K) J=\bar{\varphi}(-K),
$$

that is $\varphi(-x)=\bar{\varphi}(x)$ for almost all $x \in \mathbb{R}$. By the implication $(i) \Rightarrow$ (ii) in Prop. 4.1 above we then have

$$
\left.\Delta_{H_{1}}^{-i s} V \Delta_{H_{1}}^{i s}\right|_{s=i / 2}=J_{H_{1}} V J_{H_{1}}
$$

(namely the map $s \in \mathbb{R} \rightarrow V_{1}(s) \equiv \Delta_{H_{1}}^{-i s} V \Delta_{H_{1}}^{i s}$ extends to a bounded weakly continuous function on the closed strip $\overline{\mathbb{S}_{1 / 2}}$, analytic in $\mathbb{S}_{1 / 2}$, such that $\left.V_{1}(i / 2)=J_{H_{1}} V J_{H_{1}}\right)$. 
So

$$
\left.T(1) \Delta^{-i s} T(-1) V T(1) \Delta^{i s} T(-1)\right|_{s=i / 2}=T(1) J T(-1) V T(1) J T(-1)
$$

namely

$$
\left.\Delta^{-i s} T(-1) V T(1) \Delta^{i s}\right|_{s=i / 2}=J T(-1) V T(1) J .
$$

Since $J T(1) J=T(-1)$ and $J V J=V$, we have

$$
\left.T\left(-e^{2 \pi s}\right) V T\left(e^{2 \pi s}\right)\right|_{s=i / 2}=T(1) V T(-1)
$$

or

$$
\left.T\left(-\left(1+e^{2 \pi s}\right)\right) V T\left(1+e^{2 \pi s}\right)\right|_{s=i / 2}=V .
$$

In other words

$$
\left.T\left(-\left(1+e^{2 \pi s}\right)\right) \varphi(K) T\left(1+e^{2 \pi s}\right)\right|_{s=i / 2}=\varphi(K)
$$

in the sense that the operator-valued function $F: a \in(1, \infty) \mapsto T(a) \varphi(K) T(-a)$ admits a bounded analytic continuation in the upper half-plane $\mathbb{S}_{\infty}$ and

$$
\left.F(z)\right|_{z=0}=\left.T(-z) \varphi(K) T(z)\right|_{z=0}=\varphi(K) .
$$

Now

$$
T(a) \varphi(K) T(-a)=\varphi(T(a) K T(-a))
$$

and

$$
\begin{aligned}
T(a) \Delta^{-i s} T(-a) & =T(a) \Delta^{-i s} T(-a) \Delta^{i s} \Delta^{-i s}=T(a) T\left(-a e^{2 \pi s}\right) \Delta^{-i s} \\
& =T\left(a\left(1-e^{2 \pi s}\right)\right) \Delta^{-i s}
\end{aligned}
$$

so, differentiating at zero w.r.t. $s$ the first and the last member, we have

$$
T(a) K T(-a)=K+a P .
$$

Thus, the operator-valued function $F: a \in(1, \infty) \mapsto \varphi(K+a P)$ admits a bounded analytic continuation in the upper half-plane $\mathbb{S}_{\infty}$, and

$$
\left.F(z)\right|_{z=0}=\left.\varphi(K+z P)\right|_{z=0}=\varphi(K) .
$$

The above arguments are reversible so the lemma is proved.

Theorem 4.5. Let $(H, T)$ be an irreducible standard pair of the Hilbert space $\mathcal{H}$, set $H_{1} \equiv T(1) H$ and let $V$ be a unitary on $\mathcal{H}$ commuting with $\Delta_{H}$.

The following are equivalent:

(i) $V H_{1} \subset H_{1}$;

(ii) $V=\varphi(K)$, where $2 \pi K=-\log \Delta_{H}$ and $\varphi$ is a symmetric inner function on the upper half-plane.

Moreover the implication ( $i i) \Rightarrow(i)$ is true also if the standard pair $(H, T)$ is reducible. 
The characterization by property (ii) looks very similar to the characterization of $\mathcal{E}(H, T)$ in [13, Thm. 2.3 and Cor. 2.4], with $H$ replaced by $H_{1}$ and the generator $P$ of $T$ replaced by $K$. Yet, it requires a quite different proof, because of the lack of positivity of $K$. The trick, as in Lemma 4.4, is to consider functions $t \mapsto \varphi(K+z P)$ and exploit the positivity of $P$ instead, see Appendix B.

Proof. ( $i i) \Rightarrow(i)$ : If $\varphi$ is a symmetric inner function on the upper half-plane, then by Cor. A.3 the operator-valued function $F: a \in(1, \infty) \mapsto \varphi(K+a P)$ admits a bounded analytic continuation in the upper half-plane $\mathbb{S}_{\infty}$, and $\left.F(z)\right|_{z=0}=\varphi(K)$. Therefore by Lemma $4.4 V=\varphi(K)$ satisfies $V H_{1} \subset H_{1}$.

The implication $(i) \Rightarrow(i i)$ is proved in Appendix B.

With $(H, T)$ a standard pair of $\mathcal{H}$, we shall denote by $\mathcal{E}_{H}\left(H_{1}\right)$ the semigroup of all unitaries on $\mathcal{H}$ such that $V H=H$ and $V H_{1} \subset H_{1}$. By Thm. 4.5, if $(H, T)$ is irreducible, $\mathcal{E}_{H}\left(H_{1}\right)$ is naturally isomorphic to the semigroup of symmetric inner functions on $\mathbb{S}_{\infty}$.

\section{Classes of Models}

5.1. The two local nets on $\mathfrak{H}_{R}$ associated with a chiral net. Let $\mathcal{A}$ be a local Möbius covariant net of von Neumann algebras on $\mathbb{R}$. There are two canonical local, boost covariant nets on $\mathfrak{H}_{R}$ associated with $\mathcal{A}$, namely the ones given by (3.3) and (3.4) with $V=1$.

In other words, $\mathcal{A}$ gives rise to two local dilation covariant nets on $\mathbb{R}^{+}$. One is $\left.\mathcal{A}\right|_{\mathbb{R}^{+}}$: the associated net on $\mathfrak{H}_{R}$ is in a KMS state at inverse temperature $\beta=2 \pi$. The second one is the dilation covariant net obtained by the translation covariant net $\mathcal{A}$ on $\mathbb{R}$ by the logarithmic change of variable: the associated net on $\mathfrak{H}_{R}$ is in a ground state.

5.2. The net associated with a KMS state on a chiral net. Let $\mathcal{A}$ be a local translation covariant net of von Neumann algebras on $\mathbb{R}$. Every KMS state $\omega$ on $\mathcal{A}$, namely every locally normal KMS state at inverse temperature $\beta$ w.r.t. translations on the $\mathrm{C}^{*}$-algebra $\overline{\cup_{a>0} \mathcal{A}(-a, a)}$, gives rise to a local, boost covariant $\mathcal{A}_{\omega}$ net on $\mathfrak{H}_{R}$ by the construction in Sect. 3.

Assuming half-line duality of $\mathcal{A}$, the net $\mathcal{A}_{\omega}$ is in a KMS state at inverse temperature $\beta$.

The KMS states for the $U(1)$-current nets are known, see [6], so we have an infinite family of nets on $\mathfrak{H}_{R}$ in a KMS state.

An infinite (possibly complete) family of KMS states for the Virasoro nets is also given in [6], providing another infinite family of nets on $\mathfrak{H}_{R}$ in a KMS state.

5.3. The semigroup and family of models associated with the $U(1)$-current. Let $\mathcal{A}^{(0)}$ be the Möbius covariant net on $\mathbb{R}$ associated with the $U(1)$-current $j$, and $\mathcal{A}^{(k)}$ the net generated by the $k$-derivative of $j$.

With $V_{0}$ a unitary on the one-particle Hilbert space $\mathcal{H}_{0}$ we denote by $\Gamma\left(V_{0}\right)$ its second quantization promotion to the Bosonic Fock space over $\mathcal{H}_{0}$. We shall refer to a unitary of the form $\Gamma\left(V_{0}\right)$ as a second quantization unitary. Similarly as in the half-space case [13, Thm. 3.6], one immediately sees that $\Gamma\left(V_{0}\right)$ belongs to $\mathcal{E}_{\delta}\left(\mathcal{A}^{(k)}\right)$ iff $V$ commutes with $K^{(k)}$ and $V H_{1} \subset H_{1}$, where $K^{(k)}$ is the generator of the dilation unitary group on the one-particle Hilbert space $\mathcal{H}_{0}^{(k)}$, and $H_{1}$ is the standard subspace associated with the interval $(1, \infty)$. By Thm. 4.5, we then have: 
Theorem 5.1. A second quantization unitary $\Gamma\left(V_{0}\right)$ belongs to $\mathcal{E}_{\delta}\left(\mathcal{A}^{(k)}\right)$ if and only if $V_{0}=\varphi\left(K^{(k)}\right)$ with $\varphi$ the boundary value of a symmetric inner function on $\mathbb{S}_{\infty}$.

Therefore, by Cor. 3.3, we have:

Corollary 5.2. For every symmetric inner function $\varphi$ on $\mathbb{S}_{\infty}$ there is a $Q F T$ local net $\mathcal{A}_{V}^{(k)}$ of von Neumann algebras on $\mathfrak{H}_{R}$ in a KMS representation at $\beta=2 \pi\left(V=\Gamma\left(\varphi\left(K^{(k)}\right)\right)\right)$.

Acknowledgements. R.L. is grateful to E. Witten for stimulating comments and D. Voiculescu for pointing out ref. [14].

Open Access This article is distributed under the terms of the Creative Commons Attribution Noncommercial License which permits any noncommercial use, distribution, and reproduction in any medium, provided the original author(s) and source are credited.

\section{Appendix A. On the $H^{\infty}$ Functional Calculus of Sz.-Nagy and Foias}

Let $\mathcal{H}$ be a Hilbert space and $T \in B(\mathcal{H})$. If $\|T\| \leq 1, T$ is called a contraction. If $T$ is a contraction and there is no direct sum decomposition $T=T_{1} \oplus T_{2}$ with $T_{2}$ unitary, one says that $T$ is completely non-unitary.

If $T$ is a completely non-unitary contraction, there is an $H^{\infty}$ functional calculus for $T$, i.e., a Banach algebra homomorphism

$$
\varphi \in \mathbb{H}^{\infty}(\mathbb{D}) \mapsto \varphi(T) \in B(\mathcal{H}),
$$

defined by

$$
\varphi(T)=\lim _{r \rightarrow 1^{-}} \varphi_{r}(T),
$$

where $\varphi_{r}(z)=\varphi(r z)$, (note that $\varphi_{r}$ is analytic in the disk of radius $1 / r$ so the usual holomorphic functional calculus applies because $\operatorname{sp}(T) \subset \overline{\mathbb{D}}$ see [14]).

Proposition A.1. Let $T(z)$ be an operator-valued analytic function on a region $\mathcal{G}$ of $\mathbb{C}$, with $T(z)$ completely non-unitary contractions on $\mathcal{H}$. Given $\varphi \in \mathbb{H}^{\infty}(\mathbb{D})$, the function $z \in \mathcal{G} \mapsto \varphi(T(z))$ is analytic in $\mathcal{G}$.

Proof. With $0<r<1$, the function $z \in \mathcal{G} \mapsto \varphi_{r}(T(z))$ is analytic in $\mathcal{G}$ by the usual holomorphic functional calculus. As $r \rightarrow 1^{-}, \varphi_{r}$ converges to $\varphi$ uniformly on $\mathbb{D}$, so $\varphi_{r}(T(z))$ converges to $\varphi(T(z))$ uniformly on $\mathbb{D}$ too by the continuity of the $H^{\infty}$ functional calculus.

A densely defined linear operator $A: D(A) \subset \mathcal{H} \rightarrow \mathcal{H}$ is accretive if $\Re(\xi, A \xi) \geq 0$ for all $\xi \in D(A)$ and maximal accretive if there is no non-trivial accretive extension of $T$ on $\mathcal{H}$.

A maximal accretive operator is closed and its spectrum is contained in the right halfplane $\Re z \geq 0$. In this case the Cayley transform $T=(A+1)(A-1)^{-1}$ is a contraction. Suppose that $T$ is completely non-unitary. Then one can define a functional calculus for $T$,

$$
\varphi(A)=\varphi_{0}\left(h^{-1}(T)\right)
$$

for every function $\varphi$ in $\mathbb{H}^{\infty}(\Re z>0)$, where $h(\lambda)=\frac{\lambda+1}{\lambda-1}$ is the Cayley map (because $\left.\varphi_{0}=\varphi \circ h \in \mathbb{H}^{\infty}(\mathbb{D})\right)$. 
A.1. Case of $K+z P$. Let $U$ be the irreducible, positive energy unitary representation of the " $a x+b$ " group with non-trivial translation group. Let $P$ and $K$ be the generators of the translation and dilation group. The operator $-i(K+z P)$ is densely defined on $D=D(P) \cap D(K)$. If $\Im z \geq 0$ the operator $-i(K+z P)$ is accretive: with $z=a+i b$ and $\xi \in D$,

$$
\Re(\xi,-i(K+z P) \xi)=\mathfrak{R}[-i(\xi, K \xi)-i a(\xi, P \xi)+b(\xi, P \xi)]=b(\xi, P \xi) \geq 0 .
$$

Denote by $A(z)$ the closure of $-i(K+z P)(\mathfrak{s} z \geq 0)$.

Lemma A.2. For every $z \in \mathbb{C}$ with $\Im z \geq 0$ :

- $-i A(z)$ is maximal accretive.

- The Cayley transform $T(z)=(A(z)+i) /(A(z)-i)$ is a completely non-unitary contraction.

- The operator valued function $z \mapsto T(z)$ is analytic on $\mathbb{S}_{\infty}$ and continuous on $\overline{\mathbb{S}_{\infty}}$.

Proof. To show the first part, note that $-i A(z)$ is accretive, so it is maximal accretive iff the range of $-i(K+z P)+i$ is dense if $\Im z>0$, so we have to show that the range of $K+z P-I$ is dense if $\Im z>0$. We may assume that $U$ is in the Schrödinger representation, namely $\mathcal{H}=L^{2}(\mathbb{R}, \mathrm{d} x), K=i \frac{d}{d x}$ and $P$ is the multiplication by $e^{x}$. Then the result follows by elementary first order linear differential equation theory.

Concerning the second part, if $T(z)$ were not completely non-unitary, then $K+z P$ would have a selfadjoint restriction on a non-zero closed subspace; this implies $P=0$ on this subspace, which is not possible as there is no non-zero translation fixed vector.

The analyticity of $T(z)$ can be checked directly.

Therefore, if $\Im z \geq 0$, we can define as above $\varphi(A(z))$ for every $\varphi \in \mathbb{H}^{\infty}\left(\mathbb{S}_{\infty}\right)$, and we have:

Corollary A.3. For every fixed $\varphi \in \mathbb{H}^{\infty}\left(\mathbb{S}_{\infty}\right)$ the map $z \mapsto \varphi(A(z))$ is analytic on $\mathbb{S}_{\infty}$ and bounded, continuous on $\overline{\mathbb{S}_{\infty}}$.

Proof. Immediate by Prop. A.1 and the above discussion.

\section{Appendix B. End of Proof of Theorem 4.5}

Proposition B.1. With the notions in Thm. 4.5, if $V$ is a unitary commuting with dilations such that $V H_{1} \subset H_{1}$, then $V=\varphi(K)$ with $\varphi \in \mathbb{H}^{\infty}\left(\mathbb{S}_{\infty}\right)$.

As already noted, since $(H, T)$ is assumed to be irreducible, $V$ must be of the form $V=\varphi(K)$ with $\varphi \in L^{\infty}(\mathbb{R})$, and we must show that $\varphi \in \mathbb{H}^{\infty}\left(\mathbb{S}_{\infty}\right)$. We prove this in a few steps.

- We may assume that $\varphi$ rapidly decreases at $\pm \infty$.

Let $j_{n}$ be a non-negative, smooth function on $\mathbb{R}$ with integral 1 and $\operatorname{supp}\left(j_{n}\right) \subset$ $[0,1 / n], n \in \mathbb{N}$. Then the sequence $\left\{j_{n}\right\}$ is an approximate unit in $L^{1}(\mathbb{R})$ and $\hat{j}_{n}$ converges weakly to 1 in $L^{\infty}(\mathbb{R})$ (in the $\sigma\left(L^{\infty}, L^{1}\right)$-topology). Set $\varphi_{n} \equiv \hat{j}_{n} \varphi$; then each $\varphi_{n}$ rapidly decreases at $\pm \infty$ and their sequence converges weakly to $\varphi$ in $L^{1}(\mathbb{R})$. Morever $\hat{j}_{n} \in \mathbb{H}^{\infty}\left(\mathbb{S}_{\infty}\right)$, so $\hat{j}_{n}(K) H_{1} \subset H_{1}$ by the proven implication $(i i) \Rightarrow(i)$ in Thm. 4.5. So $\varphi_{n}(K) H_{1} \subset H_{1}$. 
It is therefore sufficient to prove the claim $\varphi_{n} \in \mathbb{H}^{\infty}\left(\mathbb{S}_{\infty}\right)$ for rapidly decreasing functions $\varphi_{n}$. Then also $\varphi \in \mathbb{H}^{\infty}\left(\mathbb{S}_{\infty}\right)$ follows, because $\varphi_{n} \rightarrow \varphi$ and $\mathbb{H}^{\infty}\left(\mathbb{S}_{\infty}\right)$ is weakly closed in $L^{\infty}(\mathbb{R})$.

- There is a dense linear space $\mathcal{D} \subset \mathcal{H}$ such that:

$\left(a_{1}\right)$ For every fixed $t>0$, the function $a \in \mathbb{R} \mapsto\left(\eta, e^{i t(K+a P)} \xi\right)$ is the boundary value of a function in $\mathbb{H}^{\infty}\left(\mathbb{S}_{\infty}\right)$, continuous in $\overline{\mathbb{S}_{\infty}}$;

$\left(a_{2}\right)$ For every fixed $z$ with $\Im z>0$, the function $t \in \mathbb{R}^{+} \mapsto\left(\eta, e^{i t(K+z P)} \xi\right)$ belongs to $L^{2}$.

Since, for a fixed $t>0$, the function $\varphi_{t}: s \mapsto e^{i t s}$ is a symmetric inner function on $\mathbb{S}_{\infty}$, by Lemma 4.4 the operator-valued map $a \in \mathbb{R} \mapsto e^{i t(K+a P)}$ is bounded analytic on $\mathbb{S}_{\infty}$, continuous in $\overline{\mathbb{S}_{\infty}}$, because $e^{i t K} H_{1} \subset H_{1}$. So the first statement $\left(a_{1}\right)$ follows.

To show $\left(a_{2}\right)$, we may work in the Schrödinger representation, so $\mathcal{H}=L^{2}(\mathbb{R}, d x)$, $e^{i t K}$ is the translation group and $P$ is the multiplication by $e^{x}$. Then

$$
\begin{aligned}
e^{i t(K+a P)} & =T(a) e^{i t K} T(-a): \\
\xi(x) \mapsto T(a) e^{i t K} e^{-i a e^{x}} \xi(x) & =T(a) e^{-i a e^{x-t}} \xi(x-t) \\
& =e^{i a e^{x}} e^{-i a e^{x-t}} \xi(x-t)=e^{i a e^{x}\left(1-e^{-t}\right)} \xi(x-t),
\end{aligned}
$$

so

$$
\left(\eta, e^{i t(K+a P)} \xi\right)=\int e^{i a e^{x}\left(1-e^{-t}\right)} \xi(x-t) \overline{\eta(x)} d x .
$$

If $\xi, \eta$ are rapidly decreasing functions we then have

$$
\begin{aligned}
\left|\left(\eta, e^{i t(K+z P)} \xi\right)\right| & \leq \int\left|e^{i z e^{x}\left(1-e^{-t}\right)} \xi(x-t) \eta(x)\right| d x=\int e^{-b e^{x}\left(1-e^{-t}\right)}|\xi(x-t) \eta(x)| d x \\
& \leq|\xi| *|\tilde{\eta}|(t),
\end{aligned}
$$

where $z=a+i b, b>0, \tilde{\eta}(t)=\eta(-t)$, and $*$ denotes the convolution product. The statement follows because $|\xi| *|\tilde{\eta}|$ is rapidly decreasing.

In the following $\varphi \in L^{\infty}(\mathbb{R})$ is rapidly decreasing at infinity, $V=\varphi(K)$ maps $H_{1}$ into itself.

- Let $f$ be the Fourier anti-transform of $\varphi$. Then $f \in L^{2}(\mathbb{R})$ and

$$
(\eta, \varphi(K) \xi)=\int_{-\infty}^{\infty} f(t)\left(\eta, e^{i t K} \xi\right) d t
$$

for all vectors $\xi, \eta$ such that $t \mapsto\left(\eta, e^{i t K} \xi\right)$ belongs to $L^{2}$, in particular for $\xi, \eta \in \mathcal{D}$.

This is obvious for all $f \in L^{1}$, and true in our case by an approximation argument.

- The map

$$
F_{+}: a \in \mathbb{R} \mapsto \int_{0}^{\infty} f(t)\left(\eta, e^{i t(K+a P)} \xi\right) d t
$$

admits a bounded analytic continuation in $\mathbb{S}_{\infty}$ for all $\xi, \eta \in \mathcal{D}$.

By the above point we may define

$$
F_{+}(z)=\int_{0}^{\infty} f(t)\left(\eta, e^{i t(K+z P)} \xi\right) d t
$$


for $\mathfrak{\Im} z>0$ providing the desired analytic continuation.

- The map

$$
F_{-}: a \in \mathbb{R} \mapsto \int_{-\infty}^{0} f(t)\left(\eta, e^{i t(K+a P)} \xi\right) d t
$$

admits a bounded analytic continuation in $\mathbb{S}_{\infty}$ for all $\xi, \eta \in \mathcal{D}$.

This is now immediate because $F_{-}=F-F_{+}$where $F(a)=(\eta, \varphi(K+a P) \xi)$ admits an analytic continuation in $\mathbb{S}_{\infty}$ because $\varphi(K) H_{1} \subset H_{1}$.

- Conclusion.

We have

$$
F_{-}(a)=\int_{-\infty}^{0} f(t)\left(\eta, e^{i t(K+a P)} \xi\right) d t=-\int_{0}^{\infty} f(-t)\left(\eta, e^{-i t(K+a P)} \xi\right) d t,
$$

and by the above argument $F_{-}$admits a bounded analytic continuation in the lower halfplane $-\mathbb{S}_{\infty}$. So $F_{-}$is constant by Liouville theorem. So $F=F_{+}$plus a constant that must be zero by $L^{2}$-integrability. This means that $\operatorname{supp}(f) \subset[0, \infty)$, so $\varphi \in \mathbb{H}^{\infty}\left(\mathbb{S}_{\infty}\right)$.

This completes the proof of Prop. B.1, and thus the conclusion $(i) \Rightarrow(i i)$ in Thm. 4.5 .

\section{References}

1. Araki, H., Zsido, L.: Extension of the structure theorem of Borchers and its application to half-sided modular inclusions. Rev. Math. Phys. 17, 491-543 (2005)

2. Borchers, H.-J.: The CPT theorem in two-dimensional theories of local observables. Commun. Math. Phys. 143, 315-332 (1992)

3. Brunetti, R., Guido, D., Longo, R.: Modular localization and Wigner particles. Rev. Math. Phys. 14, 759-785 (2002)

4. Brunetti, R., Fredenhagen, K., Verch, R.: The generally covariant locality principle: a new paradigm for local quantum field theory. Commun. Math. Phys. 237, 31-68 (2003)

5. Camassa, P., Longo, R., Tanimoto, Y., Weiner, M.: Thermal states in conformal QFT. I, to appear in Commun. Math. Phys. doi:10.1007/s00220-011-1337-3; http://arxiv.org/abs/1101.2865

6. Camassa, P., Longo, R., Tanimoto, Y., Weiner, M.: Thermal states in conformal QFT. II. http://arxiv.org/ abs/1109.2064

7. Kawahigashi, Y., Longo, R.: Classification of two-dimensional local conformal nets with $c<1$ and 2-cohomology vanishing for tensor categories. Commun. Math. Phys. 244, 63-97 (2004)

8. Kawahigashi, Y., Longo, R., Müger, M.: Multi-interval subfactors and modularity of representations in conformal field theory. Commun. Math. Phys. 219, 631-669 (2001)

9. Longo, R.: "Lectures on Conformal Nets". Preliminary lecture notes are available at http://www.mat. uniroma2.it/ longo; the first part is published as follows: Longo, R.: Real Hilbert subspaces, modular theory, $S L(2, \mathbb{R})$ and CFT. In: "Von Neumann algebras in Sibiu", Bucharest: Theta, Foundation, 2008, pp. 33-91

10. Longo, R., Rehren, K.-H.: Nets of subfactors. Rev. Math. Phys. 7, 567-597 (1995)

11. Longo, R., Rehren, K.-H.: Local fields in boundary CFT. Rev. Math. Phys. 16, 909-960 (2004)

12. Longo, R., Rehren, K.H.: How to remove the boundary in CFT, an operator algebraic procedure. Commun. Math. Phys. 285, 1165-1182 (2009)

13. Longo, R., Witten, E.: An algebraic construction of boundary QFT. Commun. Math. Phys. 303, 213$232(2011)$

14. Sz.-Nagy, B., Foias, C.: Harmonic Analysis of Operators on Hilbert Space. North-Holland, AmsterdamLondon (1970) 